\title{
Harmonic Rule for Measuring the Facial Similarities among Relatives
}

\author{
${ }^{1}$ Ravi Kumar Y B and ${ }^{2} \mathrm{C}$ N Ravi Kumar \\ ${ }^{12}$ Department of Computer Science and Engineering, Sri Jayachamarajendra College of Engineering, VTU \\ Belgaum Karnataka, India; \\ ravikumaryb@gmail.com; kumarcnr.sjce@gmail.com
}

\begin{abstract}
The harmonic rule is a strategy used to measure the harmonic distance between any pair of images. The images have been trained and tested on individual pair of facial images respectively. The facial images have been trained in five folds with equal number of facial images, where each fold consists of some equal number of images of both KinfaceW-I and KinfaceW-II, which is a benchmark dataset. The facial similarities among relatives are measured by employing a harmonic distance metric using K-nearest neighbors over a dataset KinfaceW. The proposed Harmonic Rule for Measuring the Facial Similarities among Relatives is a method used to determine the percentage of facial similarity between father-son, father-daughter, mother-son and mother-daughter. Also the proposed Extended Harmonic Rule for Measuring the Facial Similarities among relatives is another strategy used to determine the percentage of face similarities between son-father-grandfather, son-father-grandmother, daughter-father-grandfather, and daughterfather-grandmother. The result of the proposed approach is better than other approaches like NRML and MNRML, which are contemporary works published.
\end{abstract}

Keywords: Facial Similarity, Harmonic Rule, Harmonic Distance Metric, Extended Harmonic Rule

\section{Introduction}

Facial similarity is an application used to determine the face of criminal based on the suspected facial features of relatives. The determination of family tree is another application, which requires information of individual persons of a family. If there is a conflict in settling the inherited property and if the identity of the claimants within a large family is an issue, then under such circumstances, measuring facial similarity is applicable. Another application involves, while building up the family tree, true authenticated family members can be determined through facial similarity measure. The Face is accounted as an important parameter for identifying the similarity among people. Face detection, face similarity, face occlusion detection are all related domains, which work on facial images of a person. The facial features include eyes, nose, mouth, ears, chin, whose structure varies from person to person. These facial features represents a person very uniquely even in case of twins. Although the twins seem similar in nature, the facial structure of their parts varies between them, such differences in facial features can be noticed from [1],[3],[4] and [6], [7],[8], [9], [10], [11], [12], [13], [14], [15], [16], [17], [18], [19], [21], [22], [23], [24], [25], [27], [29], [30]with the help of facial feature descriptors and facial feature discriminators. 
Ravi Kumar Y B and C N Ravi Kumar; Harmonic Rule for Measuring the Facial Similarities among Relatives. Transactions on Machine Learning and Artificial Intelligence, Volume 4 No 6 December (2016); pp: 29-49

The facial feature descriptors like Local Binary Patterns (LBP), Local Ternary Patterns (LTP), Binary Rotation Invariant Scalable Keys (BRISK), Speeded-Up Robust Features (SURF), and Scale Invariant Feature Transform (SIFT) plays a vital role in understanding and analyzing the patterns of a facial structure. All these are facial feature descriptors, which provide suitable facial information of a facial image. The facial features are then extracted using principal component analysis and compared by using Harmonic metric rule to identify the distance between two or more facial features. The objective behind this approach is to determine the facial similarities among relatives and to develop an algorithm, which performs the task of determining the facial similarities among relatives. Thus, a model Harmonic Rule for Measuring Facial Similarities among Relatives (HRMFS) and Extended Harmonic Rule for Measuring the Facial Similarities among generation of Relatives (EHRMFS) has been proposed.

The proposed methods have two variants one is Harmonic Rule for Measuring Facial Similarities (HRMFS) among parents and their children and the other is Extended Harmonic Rule for Measuring Facial Similarities (EHRMFS) among children and their ancestors. In this work, measurements are done for the purpose of identifying the facial similarities among relatives with their ancestors like father and grandfather with son, mother and grand-father against son, daughter is measured with father and Grand-Father and daughter is measured with mother and Grand-Father and all possible combinations of these relations.

While measuring the similarities between two facial images, the contribution of each component of the principal component towards the mean of respective row is measured using Harmonic Mean. The harmonic rule makes use of the effectiveness of harmonic mean by measuring the mean of two images with respect to a row vector of an image. The proposed approach gives better results than other methods as indicated in table 1 and table 2 of section 6 , which discusses results of experimentation.

Section 2 of this work discusses the published papers related to facial similarities. Section 3 presents a dataset KinfaceW-I and KinfaceW-II on which the work is carried out. Section 4 introduces the proposed method and its working principle on a benchmark dataset. Section 5 discusses the experiments conducted. Section 6 gives the experimental results and its analysis.

\section{Related Work}

This section throws light on various earlier approaches employed for finding the facial similarity with its merits, demerits and results. NRML of Lu. et.al [1] describes as to how facial similarities is to be measured among relatives by employing a Euclidean distance metric, and states that the nearest samples are pushed as close as possible and other samples are pulled as far as possible is the merit of this approach and while employing this strategy, the weights assigned to individual samples is quite a difficult task, which is a limitation of this approach. A key point detector [3] gives an insight of description and processing of matching of similar facial features. G. Kaminski et al states the key point determination from the facial image is quite a good methodology used to find the facial similarity among relatives, but faces a drawback of processing of key point descriptors, which is a limitation of this approach. The kin selective behaviors [4] are more relevant to degree of relatedness among relatives, which describes the percentage of facial similarity between two or more images based on feature matching and its selection and faces limitations of measuring the relatedness of kinship. H.Bay.et.al states that the scale and rotation-invariant facial feature descriptors [5] and the interest points are detected using speeded-up robust features has an advantage of robustness of measuring the facial kinship inspite of scaling and rotation invariant situations. F. T.et.al describes the local binary patterns [6] and its use in understanding the texture of an image, The 
J.M. Morel.et.al states Scale Invariant Feature Transform [7] is a feature matching algorithm, which does the task of facial similarity estimations under scale invariant conditions. The D.Maturana.et.al discriminative learning approach [8] of measuring the facial recognition, which is a part of this paper in context of measuring the facial similarity among relatives, The $\mathrm{S}$. Hegenbart.et.al describes local ternary patterns [9] helps in understanding the facial similarity in the context of adaptive elliptic shaped neighborhoods, The B.Sheta.et.al's concept of matching the set of features [10] among a pair of images is discussed.

Baochang Zhang.et.al describes the high order local pattern descriptor [11] and its role in understanding the image has been a concept of interest. The Min Xu.et.al's insight of positive samples and its related score in kinship [12] is discussed and has been used to measure the similarity between any pair of images in our proposed work. Adaptive methods of Z. Guo et.al describes Local ternary patterns [13] as the method of learning the facial kinship measurements on faces, The S.Jegelka.et.al of BRISK features [14] and its functionality in understanding the facial images have been selected, incorporated and measured to identify the percentage of facial similarities among relatives. This BRISK provides a platform for our proposed work to understand the facial similarities among their relatives. The M.Franceschelli.et.al's estimation of Eigen values [15] and its involvement in the laplacian matrix has been understood and incorporated its usage in this proposed methods to overcome the limitations and drawbacks of other approaches in the field of measuring facial kinship. The H.Yan.et.al's facial features are to be extracted and used in discriminating the facial features [16] and to identify the facial similarities. The B.W.Dickinson.et.al Eigen vectors and its importance in recognizing [17] the facial features has been adopted in different phases of proposed algorithm and its limitations has been addressed using our proposed method. The L.Nanni.et.al describes discriminate rotation invariant pattern in terms of local binary or ternary patterns [18] and have been used in our proposed method to identify the percentage of facial similarities. The speeded up robust features [19] and its role in understanding the facial similarities in terms of descriptions have been used in our work to improve the accuracy of our proposed work. The technique of Eigen faces [20][21][22][23] has been used in the context of face recognition, which is used in this proposed method and the limitations of this work [20] has been overcome in our proposed by incorporating facial feature descriptors. The S.Xia.et.al's transfer subspace learning algorithm [24] to detect kinship among relatives is the main intuition of this, which is considered for understanding the facial similarity in our proposed work. The J.Hu.et.al's large margin multi metric learning [25] LMNN is approach gives information as to how distance metrics are used and its correlations of different feature represents, such unique property that is understood by incorporating the feature in our proposed method. The David G. Lowe.et.al describes that the prototype based discriminative feature learning [26] on facial features provides sufficient information as to how the work is to be carried out in our algorithm. The H.Yan.et.al proposed the rotation invariant using LBP variance [27] is used in this proposed method to understand the facial similarity among relatives and has been indicated in Figure3. The kinship verification under uncontrolled conditions [28] and its importance of using the mathematical model is understood while designing our algorithm. The technique [29] which is more suitable for determining the texture features and its enhancement in face recognition is considered in Eigen vector of proposed method. The triangular or three metric similarity learning [30] and its ideas have been adopted in this proposed work during the stage of measuring distance metric learning. 


\section{Datasets}

The research work has been carried out on highly standard datasets namely KinfaceW, which consists of two folders of KinfaceW-I and KinfaceW-II. The Kinface W-I consists of father-daughter, father-son, mother-daughter and mother-son data images. Similarly, KinfaceW-Il dataset consists of all possible combinations of father, son, mother and daughter data images.

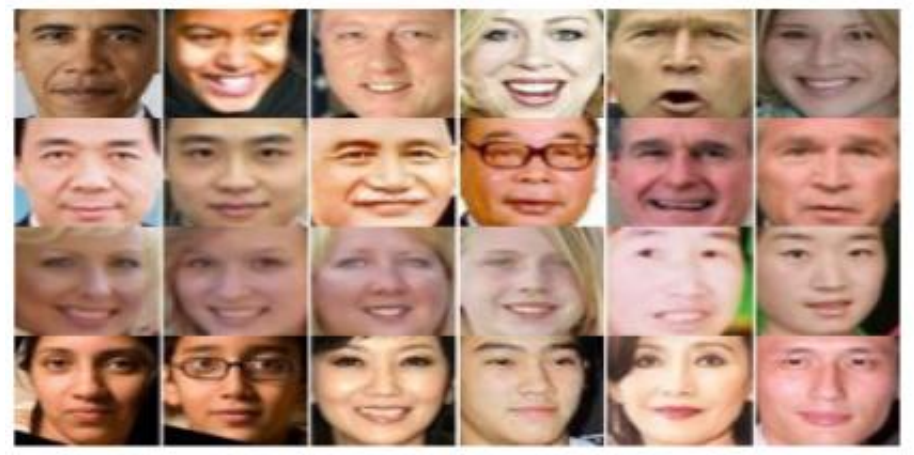

Figure 1. The dataset KinfaceW-I consisting of 3 pairs of father-daughter (FD) along row 1, 3 pairs of FatherSon (FS) along row 2, 3 pairs of Mother-Daughter (MD) along row 3, 3 pairs of Mother-Son (MS) along row4.
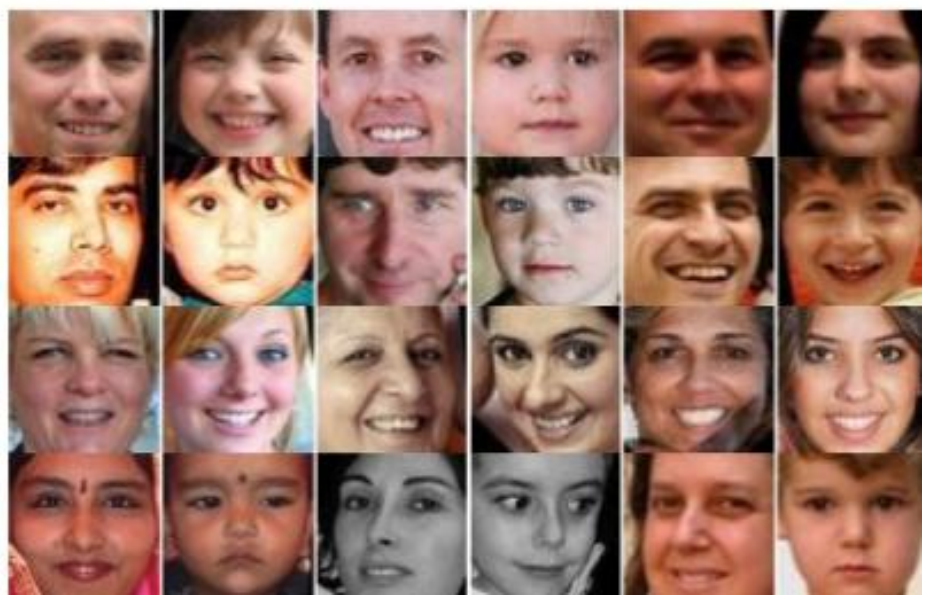

Figure 2. The dataset KinfaceW-II consisting of father-daughter (FD), Father-Son (FS), Mother-Daughter (MD), Mother-Son (MS) relationship along row 1 to row 4 respectively.

\section{Proposed Method}

The proposed method makes use of harmonic mean and its effectiveness has been realized and incorporated to improve the accuracy of the previously carried out work namely NRML and MNRML. The work involves measuring the facial similarities between two images and its percentage of similarities have been well measured by using harmonic distance metric. This section addresses the superiority of using HRMFS and EHRMFS over a dataset KinfaceW consisting of KinfaceW-I and KinfaceW-II with respect to other approaches like NRML and MNRML.

\subsection{Background Idea}

The HRMFS and EHRMFS have been designed to investigate and outperform other contemporary approaches like NRML and MNRML related to measuring the facial similarities among relatives. The 
relationship between two facial images may be a Father-Son, Father-Daughter, Mother-Son and MotherDaughter, but the objective is to measure the percentage of facial similarity and to improve the accuracy of measuring the facial similarities among relatives represented by an image. Let the given image is of size $\mathrm{mxn}$, whose matrix is represented by $\mathrm{G}$ as in (1), and $\beta_{1}, \beta_{2}, \ldots \quad \beta_{m}$ represents the successive elements of a row vector of a matrix G. Similarly, $\alpha_{1}, \alpha_{2}, \ldots \quad \alpha_{n}$ represents the successive column vector of a matrix G. By calculating $\alpha_{1}, \alpha_{2}, \ldots \quad \alpha_{n}$ and $\beta_{1}, \beta_{2}, \ldots, \beta_{m}$ the contribution of individual elements of a principal component can be understood with respect every individual images of a dataset. Thus the contribution of $\mathrm{a}_{11}$ in $\beta_{1}$ and $\alpha_{1}$, contribution of $\mathrm{a}_{22}$ in $\beta_{2}$ and $\alpha_{2}$, and so on till contribution of $\mathrm{a}_{\mathrm{mn}}$ in $\beta_{m}$ and $\alpha_{n}$ can be recognized by tracing through the principal diagonal elements of a matrix $\mathrm{G}$.

$$
\begin{gathered}
\mathrm{G}=\left[\begin{array}{ccc}
\mathrm{a}_{11} & \cdots & \mathrm{a}_{1, \mathrm{n}} \\
\vdots & \ddots & \vdots \\
\mathrm{a}_{\mathrm{m}, 1} & \cdots & \mathrm{a}_{\mathrm{m}, \mathrm{n}}
\end{array}\right] \begin{array}{r}
\beta_{1} \\
\beta_{2} \\
\beta_{\mathrm{m}}
\end{array} \\
\alpha_{1} \quad \alpha_{2} \quad \alpha_{3} \\
L=\left[\begin{array}{lll}
\alpha_{1} & \ldots & \alpha_{n}
\end{array}\right] \\
B=\left[\begin{array}{lll}
\beta_{1} & \ldots & \beta_{m}
\end{array}\right]
\end{gathered}
$$

Equation (4) represents the sum of each row of a matrix $\mathrm{G}$, where $\beta_{1}, \beta_{2}, \ldots \quad \beta_{m}$ is a coefficient matrix of a 2-Dimensional vector $G$ and (5) represents the sum of each column of a matrix $G$, where $\alpha_{1}, \alpha_{2}, \ldots \quad \alpha_{n}$ is a coefficient matrix of a 2-Dimensional vector $\mathrm{G}$. The contribution of $\mathrm{a}_{11}, \mathrm{a}_{22} \ldots \mathrm{a}_{\mathrm{mn}}$ is represented by evaluating (6).

$$
\begin{aligned}
& \beta_{i}=\sum_{i=1}^{m} \sum_{k=1}^{p} G_{i, k} \\
& \alpha_{j}=\sum_{j=1}^{n} \sum_{k=1}^{q} G_{k, j} \\
& X_{i, j}=\sum_{i=j}^{m n} \alpha_{j} \beta_{i}
\end{aligned}
$$

Where, $X_{i, j}$ is a primary diagonal element, which signifies the contribution of individual elements of a principal component of a matrix $G$.

The proposed method adopts the technique of Harmonic mean over a dataset Kinface $W$ and the characteristic of harmonic mean has helped us in improving the results as such it outperforms the other contemporary methods over a trained images. Let $\mathrm{T}=\left\{\left(x_{i}^{a}, x_{i}^{b}, y_{i}\right) \mid \mathrm{i}=1 \ldots \mathrm{M}\right\}$ is a triplet consisting of trained images of size $\mathrm{mxn}$, and $\mathrm{y}_{\mathrm{i}}$ is the label assigned to the image $x_{i}^{a}$ and $x_{i}^{b}$. The objective of this proposed approach is to employ a distance metric, which determines the closeness in similarity between any pair of two images of a dataset. The features considered may be the color of a skin or a local binary patterns or a combination of these two approaches to measure the percentage of facial similarity that exist between any pair of two images. The skin color is approximated by using either a Gabor feature descriptors or texture feature descriptors like LBP or a combination of both is used to measure the facial similarities among relatives. 
The distance metric, which estimates the pattern of facial similarity and makes the algorithm robust are indicated. We are using a harmonic distance metric, which outperforms the results of distance measure obtained by using Euclidean distance measure in other contemporary approaches. The harmonic distance metric $d$ is obtained from (7).

$$
d=\left(\left[\sum_{i=1}^{M} x_{i}^{a}\left(\frac{\hat{\mu}}{x_{i}^{a}}-1\right)^{2}-x_{i}^{b}\left(\frac{\hat{\mu}}{x_{i}^{b}}-1\right)^{2}\right]^{T} G\left[\sum_{i=1}^{M} x_{i}^{a}\left(\frac{\hat{\mu}}{x_{i}^{a}}-1\right)^{2}-x_{i}^{b}\left(\frac{\hat{\mu}}{x_{i}^{b}}-1\right)^{2}\right]\right)
$$

The distance metric $d$ of (4) is used to measure the percentage of facial similarity between two images of same pair. Thus, the matrix $\mathrm{G}$ is used with a harmonic distance metric as in (7), which can also be expressed as (8) in terms of $Z_{i}$ and $\widehat{\mu}$ of (9) and (10) respectively.

$$
\begin{gathered}
d=\sum_{i=1}^{n} x_{i}^{a} z_{i}^{a}-x_{i}^{b} z_{i}^{b} \\
Z_{i}=\left(\frac{\hat{\mu}}{x_{i}}-1\right)^{2} \\
\widehat{\mu}_{\mu}=\frac{N}{\sum_{i=1}^{n} \frac{1}{x_{i}}}
\end{gathered}
$$

The triplet $d\left(x_{i}^{a}, x_{i}^{b}, y_{i}\right)$ has been used to indicate that the two images $\left(x_{i}^{a}, x_{i}^{b}\right)$ are from the same pair of image dataset, which is used to identify the similarity between parent and child of $i^{\text {th }}$ pair labelled by $y_{i}$. The parent and child of same pairs are indicated by a label $y_{i}$. The Harmonic distance metric $d$ is a optimized solution, which could be formulated as indicated in (11), and can also be expanded to the form of (12). Further (12) can be simplified to the form of (13) to avoid the complexity of using more parameters.

$$
\begin{gathered}
\max _{G} H(G)=H_{1}(G)+H_{2}(G)-H_{3}(G) \\
=\frac{1}{M p} \sum_{i=1}^{M} \sum_{t_{1}=1}^{p} d\left(x_{i}^{a}, x_{i t_{1}}^{b}\right)+\frac{1}{M p} \sum_{i=1}^{M} \sum_{t_{2}=1}^{p} d\left(x_{i t_{2}}^{a}, x_{i}^{b}\right)-\frac{1}{M} \sum_{i=1}^{M} d\left(x_{i}^{a}, x_{i}^{b}\right) \\
\left.\left[\frac{1}{M p}\left\{\sum_{i=1}^{M} \sum_{\mathrm{t}_{1}=1}^{\mathrm{p}} \mathrm{x}_{\mathrm{i}}^{\mathrm{a}}\left(\frac{\hat{\mu}}{\mathrm{x}_{\mathrm{i}}^{\mathrm{a}}}-1\right)^{2}-\mathrm{x}_{\mathrm{it}_{1}}^{\mathrm{b}}\left(\frac{\hat{\mu}}{\mathrm{x}_{\mathrm{it}}^{\mathrm{b}}}-1\right)^{\mathrm{b}}\right\}^{\mathrm{T}} \mathrm{G}\left\{\sum_{\mathrm{i}=1}^{\mathrm{T}} \sum_{\mathrm{t}_{1}=1}^{\mathrm{p}} \mathrm{x}_{\mathrm{i}}^{\mathrm{a}}\left(\frac{\hat{\mu}}{\mathrm{x}_{1}^{\mathrm{a}}}-1\right)^{2}-\mathrm{x}_{\mathrm{t}_{1}}^{\mathrm{b}}\left(\frac{\hat{\mu}}{\mathrm{x}_{\mathrm{it}}^{\mathrm{b}}}-1\right)^{\mathrm{b}}\right\}\right)\right] \\
\left.+\left\{\sum_{\mathrm{i}=1}^{\mathrm{M}} \sum_{\mathrm{t}_{2}=1}^{\mathrm{p}} \mathrm{x}_{\mathrm{it}_{2}}^{\mathrm{a}}\left(\frac{\hat{\mu}}{\mathrm{x}_{\mathrm{it}_{2}}^{\mathrm{a}}}-1\right)^{2}-\mathrm{x}_{\mathrm{i}}^{\mathrm{b}}\left(\frac{\hat{\mu}}{\mathrm{x}_{\mathrm{i}}^{\mathrm{b}}}-1\right)^{2}\right\}^{\mathrm{T}} \mathrm{G}\left\{\sum_{\mathrm{i}=1}^{\mathrm{M}} \sum_{\mathrm{t}_{2}=1}^{\mathrm{p}} \mathrm{x}_{\mathrm{it}_{2}}^{\mathrm{a}}\left(\frac{\hat{\mu}}{\mathrm{x}_{\mathrm{it}_{2}}^{\mathrm{a}}}-1\right)^{2}-\mathrm{x}_{\mathrm{i}}^{\mathrm{b}}\left(\frac{\hat{\mu}}{\mathrm{x}_{\mathrm{i}}^{\mathrm{b}}}-1\right)^{2}\right\}\right] \\
-\left[\frac{1}{\mathrm{M}} \sum_{\mathrm{i}=1}^{\mathrm{M}} \mathrm{x}_{\mathrm{i}}^{\mathrm{a}}\left(\frac{\hat{\mu}}{\mathrm{x}_{\mathrm{i}}^{\mathrm{a}}}-1\right)^{2}-\mathrm{x}_{\mathrm{i}}^{\mathrm{b}}\left(\frac{\hat{\mu}}{\mathrm{x}_{\mathrm{i}}^{\mathrm{b}}}-1\right)^{2}\right]
\end{gathered}
$$


Where, $x_{i t_{1}}^{a}$ is a k-nearest neighbor of $x_{i}^{a}$ in feature vector space $\mathrm{t}_{1}$ and $x_{i t_{2}}^{b}$ is the k-nearest neighbor of $x_{i}^{b}$ in $t_{2}$ feature vector space. Equation (12) measures the closeness between two $x_{i}^{a}$ and $x_{i}^{b}$ and any similarity measure must satisfy these four properties like positivity, symmetry, sub-additives, and identity of discernible or triangular inequality. If a similarity measure of (13) satisfies these four properties, then we say that the distance metric adopted is positive, symmetric, and semi-definite. Thus we have solved the problem of facial similarity measure using iterative approach. As the approach is being used to measure the percentage of facial similarity by the way of harmonic mean as a distance metric, we require few parameters like $x_{i}^{a}$ and $x_{i}^{b}$, whose closeness could be measured with respect to K-nearest neighbor approach.

Since matrix $\mathrm{G}$ is positive, symmetric, and semi-definite, we can define it in terms of a vector $\mathrm{W}$ of size $m \times n$, where $m \geq n$ and the corresponding vector can be written in the form of (14).

$$
G=W W^{T}
$$

By adding (1) and (14), we obtain (15), which is the simplified form of (11).

$$
\begin{gathered}
H_{1}(G)=\left[\frac { 1 } { M p } ( \sum _ { i = 1 } ^ { M } \sum _ { t _ { 1 } = 1 } ^ { p } x _ { i } ^ { a } ( \frac { \hat { \mu } } { x _ { i } ^ { a } } - 1 ) ^ { 2 } - x _ { i t _ { 1 } } ^ { b } ( \frac { \hat { \mu } } { x _ { i t _ { 1 } } ^ { b } } - 1 ) ^ { 2 } ) ^ { T } W W ^ { T } \left(\sum_{i=1}^{M} \sum_{t_{1}=1}^{p} x_{i}^{a}\left(\frac{\hat{\mu}}{x_{i}^{a}}-1\right)^{2}\right.\right. \\
\left.\left.-x_{i t_{1}}^{b}\left(\frac{\hat{\mu}}{x_{i t_{1}}^{b}}-1\right)^{2}\right)\right] \\
=\operatorname{tr}\left[\frac { 1 } { M p } W ^ { T } ( \sum _ { i = 1 } ^ { M } \sum _ { t _ { 1 } = 1 } ^ { p } x _ { i } ^ { a } ( \frac { \hat { \mu } } { x _ { i } ^ { a } } - 1 ) ^ { 2 } - x _ { i t _ { 1 } } ^ { b } ( \frac { \hat { \mu } } { x _ { i t _ { 1 } } ^ { b } } - 1 ) ^ { 2 } ) ^ { T } \left(\sum_{i=1}^{M} \sum_{t_{1}=1}^{p} x_{i}^{a}\left(\frac{\hat{\mu}}{x_{i}^{a}}-1\right)^{2}-\right.\right. \\
\left.\left.x_{i t_{1}}^{b}\left(\frac{\hat{\mu}}{x_{i t_{1}}^{b}}-1\right)^{2}\right) W\right] \\
=t r\left[W^{T} K_{1} W\right] \\
\text { Where, } k_{1}=\operatorname{tr}\left[\frac { 1 } { \mathrm { Mp } } \mathrm { W } ^ { \mathrm { T } } ( \sum _ { \mathrm { i } = 1 } ^ { \mathrm { M } } \sum _ { \mathrm { t } _ { 1 } = 1 } ^ { \mathrm { p } } \mathrm { x } _ { \mathrm { i } } ^ { \mathrm { a } } ( \frac { \hat { \mu } } { \mathrm { x } _ { \mathrm { i } } ^ { \mathrm { a } } } - 1 ) ^ { 2 } - \mathrm { x } _ { \mathrm { it } _ { 1 } } ^ { \mathrm { b } } ( \frac { \hat { \mu } } { \mathrm { x } _ { \mathrm { it } } \mathrm { b } } - 1 ) ^ { 2 } ) ^ { \mathrm { T } } \left(\sum_{\mathrm{i}=1}^{\mathrm{M}} \sum_{\mathrm{t}_{1}=1}^{\mathrm{p}} \mathrm{x}_{\mathrm{i}}^{\mathrm{a}}\left(\frac{\hat{\mu}}{\mathrm{x}_{\mathrm{i}}^{\mathrm{a}}}-1\right)^{2}-\right.\right. \\
\left.\left.\mathrm{x}_{\mathrm{it}}^{\mathrm{b}}\left(\frac{\hat{\mu}}{\mathrm{x}_{\mathrm{it}}}-1\right)^{2}\right) \mathrm{~W}\right]
\end{gathered}
$$

Similarly, $H_{2}(G)$ and $H_{3}(G)$ can be written in simplified form as (19) and (23). 


$$
\begin{aligned}
H_{2}(G)= & {\left[\frac { 1 } { M p } ( \sum _ { i = 1 } ^ { M } \sum _ { t _ { 2 } = 1 } ^ { p } x _ { i _ { 2 } } ^ { a } ( \frac { \hat { \mu } } { x _ { i _ { 2 } } ^ { a } } - 1 ) ^ { 2 } - x _ { i } ^ { b } ( \frac { \hat { \mu } } { x _ { i } ^ { b } } - 1 ) ^ { 2 } ) ^ { T } W ^ { T } \left(\sum_{i=1}^{M} \sum_{t_{2}=1}^{p} x_{i t_{2}}^{a}\left(\frac{\hat{\mu}}{x_{i_{2}}^{a}}-1\right)^{2}\right.\right.} \\
& \left.\left.-x_{i}^{b}\left(\frac{\hat{\mu}}{x_{i}^{b}}-1\right)^{2}\right)\right] \\
& -x_{i}^{b}\left(\frac { 1 } { x _ { i } ^ { b } } W ^ { T } ( \sum _ { i = 1 } ^ { M } \sum _ { t _ { 2 } = 1 } ^ { p } x _ { i _ { 2 } } ^ { a } ( \frac { \hat { \mu } } { x _ { i t _ { 2 } } ^ { a } } - 1 ) ^ { 2 } - x _ { i } ^ { b } ( \frac { \hat { \mu } } { x _ { i } ^ { b } } - 1 ) ^ { 2 } ) ^ { T } \left(\sum_{i=1}^{M} \sum_{t_{2}=1}^{p} x_{i_{2}}^{a}\left(\frac{\hat{\mu}}{x_{i_{2}}^{a}}-1\right)^{2}\right.\right. \\
& \left.\left.-1)^{2}\right)_{W}\right]
\end{aligned}
$$

Where, $\quad \mathrm{k}_{2} \triangleq \operatorname{tr}\left[\frac{1}{\mathrm{Mp}} \mathrm{W}^{\mathrm{T}}\left(\sum_{\mathrm{i}=1}^{\mathrm{M}} \sum_{\mathrm{t}_{2}=1}^{\mathrm{p}} \mathrm{x}_{\mathrm{it}_{2}}^{\mathrm{a}}\left(\frac{\hat{\mu}}{\mathrm{x}_{\mathrm{it}_{2}}^{\mathrm{a}}}-1\right)^{2}-\mathrm{x}_{\mathrm{i}}^{\mathrm{b}}\left(\frac{\hat{\mathrm{\mu}}}{\mathrm{x}_{\mathrm{i}}^{\mathrm{b}}}-1\right)^{2}\right)^{\mathrm{T}}\left(\sum_{\mathrm{i}=1}^{\mathrm{M}} \sum_{\mathrm{t}_{2}=1}^{\mathrm{p}} \mathrm{x}_{\mathrm{it}_{2}}^{\mathrm{a}}\left(\frac{\hat{\mu}}{\mathrm{x}_{\mathrm{it}_{2}}^{\mathrm{a}}}-1\right)^{2}-\right.\right.$ $\left.\left.x_{i}^{b}\left(\frac{\hat{\mu}}{x_{i}^{b}}-1\right)^{2}\right) W\right]$

$$
\begin{array}{r}
H_{3}(G)=\operatorname{tr}\left[W^{T} \frac{1}{M} \sum_{i=1}^{m} x_{i}^{a}\left(\frac{\widehat{\mu}}{x_{i}^{a}}-1\right)^{2}-x_{i}^{b}\left(\frac{\widehat{\mu}}{x_{i}^{b}} 1\right)^{2} W\right] \\
\text { Where, } \mathrm{K}_{3} \triangleq \operatorname{tr}\left[\frac{1}{\mathrm{M}} \sum_{\mathrm{i}=1}^{\mathrm{M}}\left(\mathrm{x}_{\mathrm{i}}^{\mathrm{a}}\left(\frac{\hat{\mu}}{\mathrm{x}_{\mathrm{i}}^{\mathrm{a}}}-1\right)^{2}-\mathrm{x}_{\mathrm{i}}^{\mathrm{b}}\left(\frac{\hat{\mu}}{\mathrm{x}_{\mathrm{i}}^{\mathrm{b}}}-1\right)^{2}\right)\right] \\
\mathrm{H}_{3}(\mathrm{G})=\operatorname{tr}\left[\mathrm{W}^{\mathrm{T}} \mathrm{K}_{3} \mathrm{~W}\right]
\end{array}
$$

Equation. (11) may be rewritten in a more simplified manner as in the form of (18)

$$
\begin{gathered}
\max _{G} H(G)=\operatorname{tr}\left[\mathrm{W}^{\mathrm{T}}\left(\mathrm{K}_{1}+\mathrm{K}_{2}-\mathrm{K}_{3}\right) \mathrm{W}\right] \\
G=B L
\end{gathered}
$$

Equation. (19) can be written as $\gamma=\alpha+\beta$, which is same as obtaining (6) from (4) and (5). Thus, we can state that the result obtained from (18) is robust and accurate in measuring the facial similarities that exists between any pair of images. The value of $\mathrm{W}$ may be obtained from the Eigen value problem as in (20) 


$$
\left(K_{1}+K_{2}-K_{3}\right) \omega=\lambda \omega
$$

After obtaining the value of $W$, we can cross verify the similarity results obtained in (6) against (19).

\section{Algorithm 1.HRMFS}

Input: Training image pairs $\mathrm{T}=\left\{\left(x_{i}^{a}, x_{i}^{b}\right) \mid \mathrm{i}=1 \ldots \mathrm{M}\right.$ and $\left.\mathrm{a}, \mathrm{b} \in \mathrm{T}\right\}$, parameters used: minimized error $\varepsilon=0.00001$.

Output: Vector $G=L+B$, where $L=\left[\alpha_{1} \ldots \alpha n\right]$ and $B=\left[\beta_{1} \ldots \beta m\right]$.

\section{Step 1: (Initialization)}

Apply distance metric $d$ using harmonic rule to determine the closeness between $x_{i}^{a}$ and $x_{i}^{b}\left(x_{i}^{a}, x_{i}^{b}\right)$.

\section{Step 2: (Optimized Solution)}

Use K-NN algorithm to find the closeness between any pair of two images iteratively. Each iteration is indicated by $\mathrm{I}$, where $\mathrm{I}=1 \ldots . \mathrm{n}$

2.1 Solve $\mathrm{H}_{1}, \mathrm{H}_{2}$, and $\mathrm{H}_{3}$ iteratively

2.2 Obtain $X_{i, j}$ as per (6), where $\mathrm{L}=\left[\alpha_{1} \ldots \alpha_{\mathrm{n}}\right]$, and $\mathrm{B}=\left[\beta_{1} \ldots \beta_{\mathrm{m}}\right]$

2.3 Obtain the contribution of $\alpha_{\mathrm{n}}$ and $\beta_{\mathrm{m}}$ using $X_{i, j}$.

2.4 Obtain the value of $X_{i, j}$ and compare the closeness between $x_{i}^{a}$ and $x_{i}^{b}$ using K-NN algorithm.

2.5 Solve Eigen value as per (28)

2.6 Update the values of $X_{i, j}$ for next iteration of $\mathrm{I}$.

2.7 If Iteration I $>2$ and $X_{i, j}$ of iteration $\mid \mathrm{I}$ and $\mathrm{I}-1 \mid<\varepsilon$, where $\varepsilon<=0.00001$, repeat go to step2.1

\section{Step 3: (Compare the Optimized Output value)}

3.1 Compare the output distance metric $X_{i, j}$ against $\mathrm{H}(\mathrm{G})$ to cross verify the output $\mathrm{G}=\mathrm{G}^{\prime}$

Algorithm.1. HRMFS is an accurate algorithm, which measures the similarity between two faces of same pair and cross verifies the result obtained in the form of (18). Since we are cross verifying the result obtained in the form of a coefficient matrix $\mathrm{G}$ against $X_{i, j}$, the term accurate is used.

The Extended Harmonic Rule is a method used for Measuring the Facial Similarities among relatives and to calculate the percentage of facial similarity not only between Father-Son(FS), Father-Daughter(FD), Mother-Son(MS), and Mother-Daughter(MD), but also to extended the functionality of measuring the percentage of facial similarities among Grandfather-Grandson(GF-GS), Grandfather-Granddaughter(GFGD), Grandmother-Grandson(GM-GS), and Grandmother-Granddaughter(GM-GD). The EHRMFS has to be even more robust than HRMFS, as we are interested to measure the similarity between ancestors and their children under varying condition like factor of age, which results in change of facial features in ancestors like wrinkles in the face of ancestors than the immediate ancestors of the children. The facial feature descriptors used in this approach remains same, but the parameter considered to estimate the facial similarity among relatives is quite different than the approach HRMFS.

As the method HRMFS has been Extended to calculate the percentage of facial similarities among the generations of facial images, we have incorporated the idea of chain rule, where the chain rule applies 
the proposed EHRMFS in the form of PQR, QRP, RPQ as such it does not violates the rule of chain. Let tr $(P), \operatorname{tr}(Q)$, and $\operatorname{tr}(R)$ be the three matrices representing the facial features of Grand-Father, Father and Son or Grand -Father, Mother and Son.

$$
\begin{aligned}
& \operatorname{tr}(P)=p_{11}+p_{22}+\cdots+p_{n n} \\
& \operatorname{tr}(Q)=q_{11}+q_{22}+\cdots+q_{n n} \\
& \operatorname{tr}(R)=r_{11}+r_{22}+\cdots+r_{n n}
\end{aligned}
$$

Where, $P, Q, R$ is a nonnegative matrix of the form

$$
\begin{gathered}
P=\left[\begin{array}{ccc}
p_{11} & \cdots & p_{1, n} \\
\vdots & \ddots & \vdots \\
p_{m, 1} & \cdots & p_{m, n}
\end{array}\right], Q=\left[\begin{array}{ccc}
q_{11} & \cdots & q_{1, n} \\
\vdots & \ddots & \vdots \\
q_{m, 1} & \cdots & q_{m, n}
\end{array}\right], R=\left[\begin{array}{ccc}
r_{11} & \cdots & r_{1, n} \\
\vdots & \ddots & \vdots \\
r_{m, n} & \cdots & r_{m, n}
\end{array}\right] \\
\operatorname{tr}(P Q R)=\operatorname{tr}(Q R P)=\operatorname{tr}(R P Q)
\end{gathered}
$$

Where, $\operatorname{tr}(P R Q) \neq \operatorname{tr}(P Q R) \neq \operatorname{tr}(R Q P)$ or any other combination, which does not satisfies the rule of chain is not acceptable, while measuring the facial similarities among generation of facial images. i.e., only the trace of sequence of nonnegative matrices is possible as per (24) and the trace of PQR may be indicated as Grandfather, Father, and Son respectively. Similarly, QRP may be indicated as Father, Son, and Grandfather, so on and so forth.

Equation (21), (22), (23) may be written in terms of $\alpha, \beta$ as in (25).

$$
\begin{aligned}
P_{i, j} & =\sum_{i=j}^{m n} \alpha_{j} \beta_{i} \\
Q_{i, j} & =\sum_{i=j}^{m n} \alpha_{j} \beta_{i} \\
R_{i, j} & =\sum_{i=j}^{m n} \alpha_{j} \beta_{i}
\end{aligned}
$$

As we measuring the facial similarities among Grandfather-Grandson(GF-GS), GrandfatherGranddaughter(GF-GD), Grandmother - Grandson (GM-GS) and Grandmother - Granddaughter (GM-GD), the problem EHRMFS may be formulated as in (26). Similarly, the similarity between two images is carried out using $\operatorname{tr}(\mathrm{PQ})=\operatorname{tr}(\mathrm{QP})$

$$
\begin{aligned}
& \underbrace{\max H(G)}_{G}=\operatorname{tr}\left[\begin{array}{c}
\underbrace{\left(H_{1}^{p}(P)+H_{2}^{p}(P)-H_{3}^{p}(P)\right)}_{P}+\underbrace{\left(H_{1}^{q}(Q)+H_{2}^{q}(Q)-H_{3}^{q}(Q)\right)}_{Q}) \\
+\underbrace{\left(H_{1}^{r}(R)+H_{2}^{r}(R)-H_{3}^{r}(R)\right)}_{R}
\end{array}\right] \\
& \operatorname{tr}(P Q R)=\operatorname{tr}(Q R P)=\operatorname{tr}(R P Q)
\end{aligned}
$$


The trace of a matrix of (38) helps in understanding the facial similarities between two or more images represented in the form of $\mathrm{G}_{1}=L_{p}+B_{p}, \mathrm{G}_{2}=L_{q}+B_{q}$ and $\mathrm{G}_{3}=L_{r}+B_{r}$. Where, $L_{p}=$ $\left[\begin{array}{lll}\alpha_{1}, & \alpha_{2}, \ldots & \alpha_{m}\end{array}\right]$ and $B_{p}=\left[\begin{array}{l}\beta_{1} \\ \beta_{2} \\ \beta_{n}\end{array}\right]$. The diagonal element of a matrix is represented by principal diagonal.

Finally, the contribution of $\mathrm{a}_{11}$ along $\alpha_{1}$ and $\beta_{1}$. Similarly, the contribution of $\mathrm{a}_{22}$ is obtained along $\alpha_{2}$ and $\beta_{2}$ so on till $\mathrm{a}_{\mathrm{mn}}$ along $\alpha_{m}$ and $\beta_{n}$. Further, the importance of HRMFS and EHRMFS can be understood with respect to the experiments conducted.

From (24), we can extract the facial features of a person, while _ represents the type of curves or wrinkles available on the face is estimated in every iteration of EHRMFS algorithm. Thus (24) can be written the form of $\beta$ like (41)

$$
\begin{gathered}
\max =\operatorname{tr}\left[H_{1}(P)+H_{2}(P)-H_{3}(P), H_{1}(Q)+H_{2}(Q)-H_{3}(Q), H_{1}(R)+H_{2}(R)-H_{3}(R)\right] \\
\max =\left[\frac{\operatorname{tr}\left(W^{T}\left(k_{1}+k_{2}-k_{3}\right) W\right)}{\sum_{i=1}^{k} \operatorname{tr}\left(W^{T}\left(k_{1}+k_{2}-k_{3}\right) W\right)}\right] \\
=[\mathrm{WT}(\mathrm{K} 1 \beta \mathrm{k}+\mathrm{K} 2 \beta \mathrm{k}-\mathrm{K} 3 \beta \mathrm{k}) \mathrm{W}
\end{gathered}
$$

Further, The below (39) and (40) are similar to (26), which can be calculated in EHRMFS. Where (38) can be further reduce to $\max (G, \beta)=\left[\mathrm{W} \beta_{\mathrm{k}}(\mathrm{K} 1+\mathrm{K} 2-\mathrm{K} 3) \mathrm{W}^{\top}\right]$. Further, the below (43) is similar to (26), which can be calculated in EHRMFS.

$$
\sum_{k=1}^{m} \beta_{k}\left[\left(k_{1}+k_{2}-k_{3}\right) \omega\right]=\lambda \omega
$$

\section{Algorithm 2: EHRMFS}

Input: Labeled image pairs $\mathrm{T}=(\mathrm{xai}, \mathrm{xbi}) \mid \mathrm{i}=1 \ldots \mathrm{M}$ and $\mathrm{a} 1, \mathrm{~b} 2 \mathrm{~T}$, parameters used: minimized error $\epsilon=0.00001$.

Description: The given input image is processed to calculate the wrinkles in face by employing a strategy of $\beta \mathrm{k}$.

Output: Determines the facial similarity between ancestors and their children.

Step1: (Initialization)

Step 1.1:Set_k $=\mathrm{i} \mid \mathrm{i}=[1 \ldots \mathrm{K}]$

Step 2: (Global Optimization of facial features)

Step 2.1: Solve $\mathrm{H} 1, \mathrm{H} 2$, and $\mathrm{H} 3$ iteratively

Step 2.2: Calculate $\beta_{\mathrm{k}}$ from (37)

Step 2.3: Obtain $\lambda$ by solving (42)

Step 2.4: Update $\beta_{\mathrm{k}}$ from next iteration

Step 2.5: If $\beta_{i}=\beta_{i-1}<\_$go to Step 3 
Ravi Kumar Y B and C N Ravi Kumar; Harmonic Rule for Measuring the Facial Similarities among Relatives. Transactions on Machine Learning and Artificial Intelligence, Volume 4 No 6 December (2016); pp: 29-49

Step 3: (Compare the optimized solution)

Step 3.1: Compare the output distance metric WG, against $W I$ to cross verify the output $G=G^{\prime}$

\section{Conduction of Experiments}

Experiments were conducted on datasets like KinfaceW -I and Kinface W-II using different facial feature descriptors for the purpose of measuring the facial similarities among relatives and to evaluate the robustness of HRMFS, and EHRMFS over different KinfaceW dataset. The experimental results produced by this approach namely HRMFS and EHRMFS can be employed on public figures, which are downloadable on social networking sites. The procedure followed for the conduction of experiments is described in the subsections.

\subsection{Procedure of experiments}

The procedure followed to conduct an experiment has been described in 3 phases, where in the phase 1 performs cropping up of images to a standard size, phase 2 extracts the facial features using different feature descriptors to obtain the description of an image, and phase 3 performs the task of training and testing of data obtained from facial feature descriptors.

Phase 1: Cropping-up of facial images

Phase 2: Facial Feature descriptors

Phase 3: training and testing of data.

\subsubsection{Cropping-up of images}

The facial regions of an image is cropped manually and resized to $128 \times 128$. So as to get only positive samples of an image in the facial feature descriptors and to ignore all the negative samples of an image. As we Extended to achieve a greater accuracy over the dataset KinFace W- I and Kinface W -II, we considered only positive samples by cropping the images manually. The aim of cropping each and every image is to obtain better results, when facial feature descriptors are applied on the image and compared with the corresponding images of the same dataset. The resizing of images to $128 \times 128$ is done to enable the descriptors to get enough information of individual samples of all the images of the dataset.

\subsubsection{Facial feature descriptors}

The facial feature descriptor is a method used to obtain the description of individually cropped images in terms of bins. In order to obtain a greater accuracy of determining the facial similarity among relatives, the descriptors have been designed in the following manner and obtained the results.

Local Binary Patterns: It is a facial feature descriptor designed to obtain the description of a facial feature so as to make an algorithm HRMFS and EHRMFS more robust. The design of LBP involves dividing each and every image into $8 \times 8$ blocks, where each block gives a histogram of an image of size 256 Dimension and these individual 256 Dimensions of image data is concatenated with other individual blocks. So as to obtain an image data of $256 \times 64$ equal to 16,384 Dimensional image data. As we resized an image to 128 $x 128$ during the cropping of an image, we were required to subdivide an image into $8 \times 8$, where each block is of size $16 \times 16$. Thus, we obtained a high dimensional data of 16,384 , which is sufficient and gives enough information of an image. 

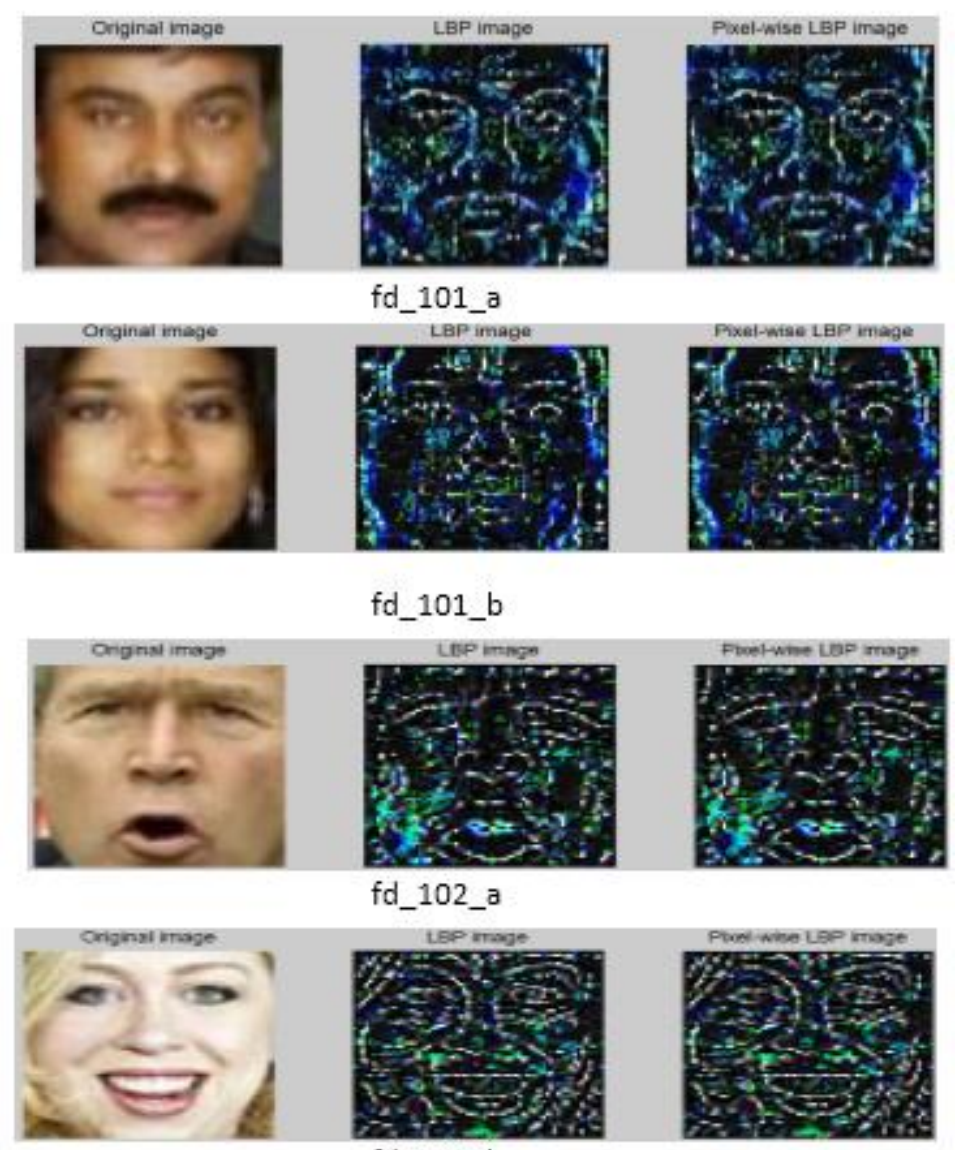

fd_102_b

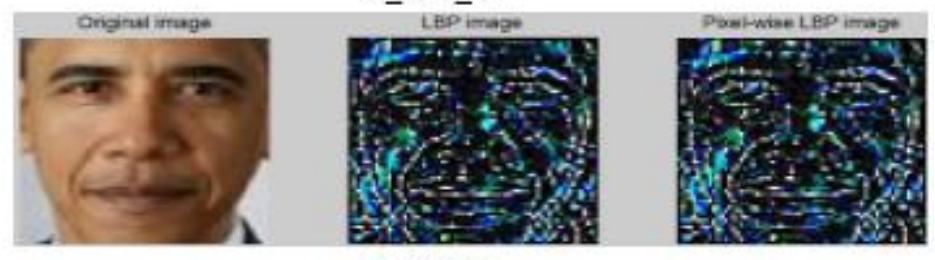

fd_103_a

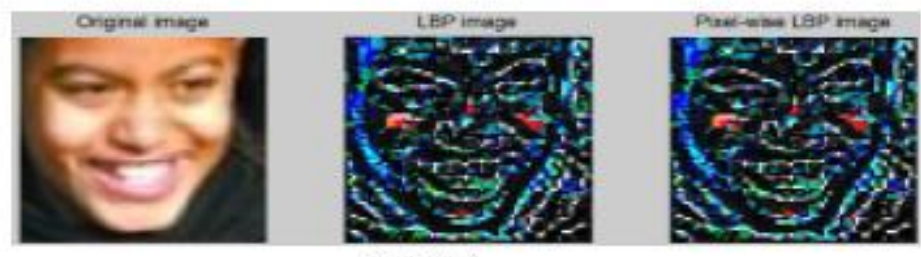

fd_103_b

Figure3. gives an output of face descriptors applied with Local binary pattern technique.

Scale Invariant Feature Transform: It is a feature descriptor designed to get more accurate and detailed information of a face. In order to find out the facial features of an image, we divided each and every facial image into non overlapping blocks of size $16 \times 16$ and extracted a facial feature of individual patches and matched with the corresponding patch of the other facial image.

Three Patch Local Binary Pattern: It is a facial feature descriptor used to obtain the facial features of an image by concatenating histograms of an individual patches of an image, where the single individual image is divided into blocks of size $16 \times 16$ and each individual patch is applied with TPLBP to obtain an histogram 
of individual blocks of an image and finally the histograms of individual patches are concatenated to get TPLBP of an image. TPLBP converges to an LBP after splitting a TPLBP histogram into two TPLBP, comprising of upper TPLBP and lower TPLBP. The result of this process leads to a more detailed information of a face.

\subsubsection{Training and testing of data}

This phase involves grouping of images of a dataset KinfaceW-I and KinfaceW-II and then the pair of images are considered for the purpose of evaluation. The result of this is a 5 fold result obtained on an image dataset. The training stage involves making the system understand the facial structure of individual images and its similarities with respect to its relatives like father, mother etc. The testing stage involves determining the facial similarities among relatives based on the information gathered from the training process.

\section{$7 \quad$ Results and Analysis}

The results of the proposed HRMFS and EHRMFS with different facial feature descriptors like LBP, SIFT, TPLBP over a KinfaceW-I and KinfaceW-II dataset is shown in different figures from Figure4, Figure5, Figure6, and Figure7. Similarly, Figure8, Figure9 and Figure10, and Figure11 respectively, where the straight line at $45^{\circ}$ is the reference line. The result above this reference line indicates the improvement of the performance in establishing the accuracy of relationship.

Results of dataset KinfaceW-I

The HRMFS and EHRMFS have been developed by incorporating the significant characteristic of harmonic metric in this proposed work and the superiority of the proposed method can be realized with reference to other approaches like NRML and MNRML. In order to understand and analyze the efficiency of the proposed method, graphs are plotted with respect to false positive rate and true positive rate

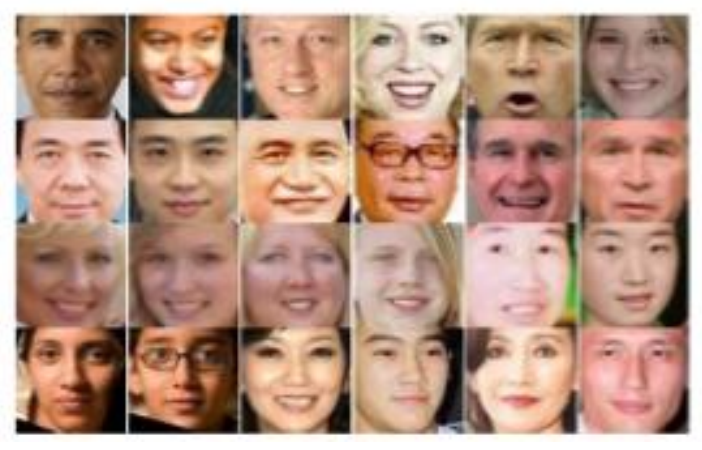

Figure 4. The first row of the dataset indicates 3 pairs of Father-Daughter relationship, Second row indicates 3 pairs of Father-Son relationship, Third row shows 3 pairs of Mother-Daughter relationship, and Last row indicates 3 pairs of Mother-Son relationship 


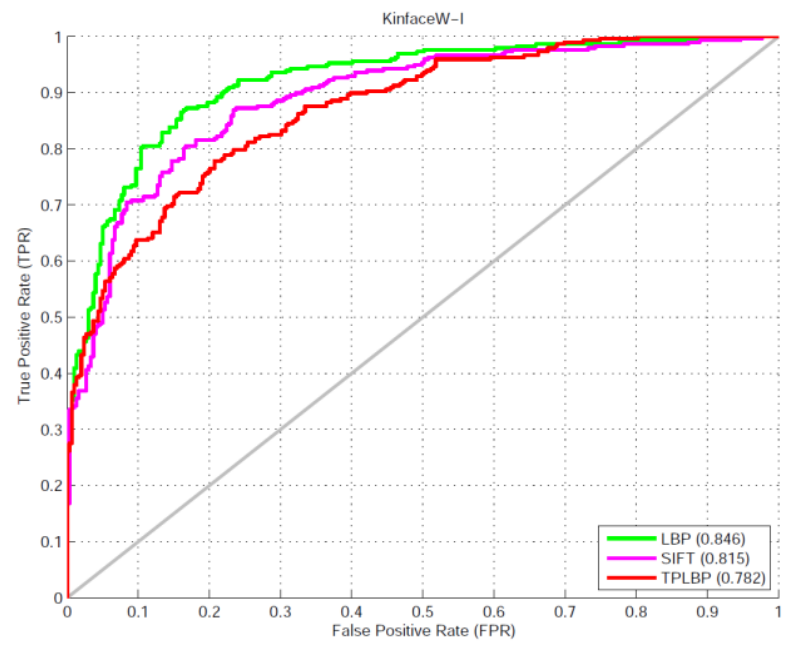

Figure 5. The result of ROC on different datasets like KinfaceW-I with different possible relationships.

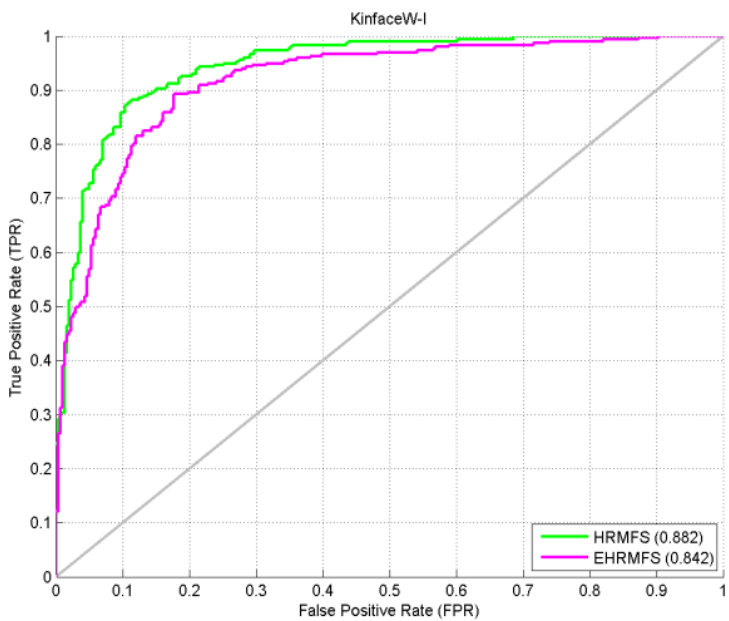

Figure 6. The accuracy of HRMFS and EHRMFS over KinfaceW-I dataset indicates the results of comparision.

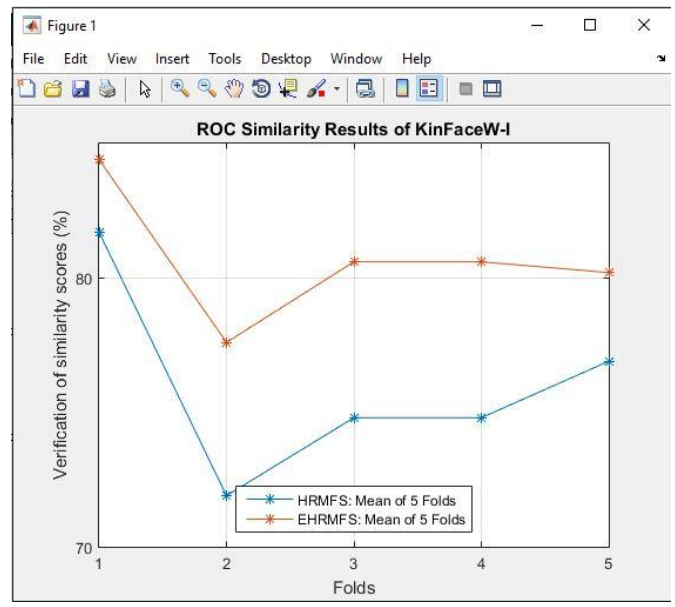

Figure 7. Indicates the mean verification accuracy of the proposed method HRMFS and EHRMFS over a dataset KinfaceW-I. 


\subsection{Results of dataset KinfaceW-II}

The HRMFS and EHRMFS can be realized and understood by referring to the graphs obtained from the proposed work and the results obtained over a dataset KinfaceW-II with different combination of relationships are shown in Figure(7), Figure(8) and Figure(9).

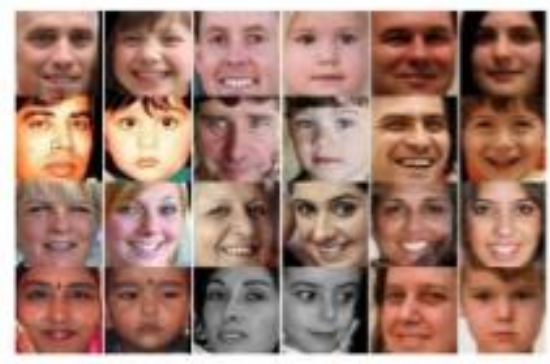

Figure 8. The dataset consisting of different relationships with 3 pairs of Father-Daughter, 3 pairs of FatherSon, 3 pairs of Mother-Daughter, and 3 pairs of Mother-Son are shown in first row, second row, third row and fourth row of the dataset KinfaceW-II respectively.

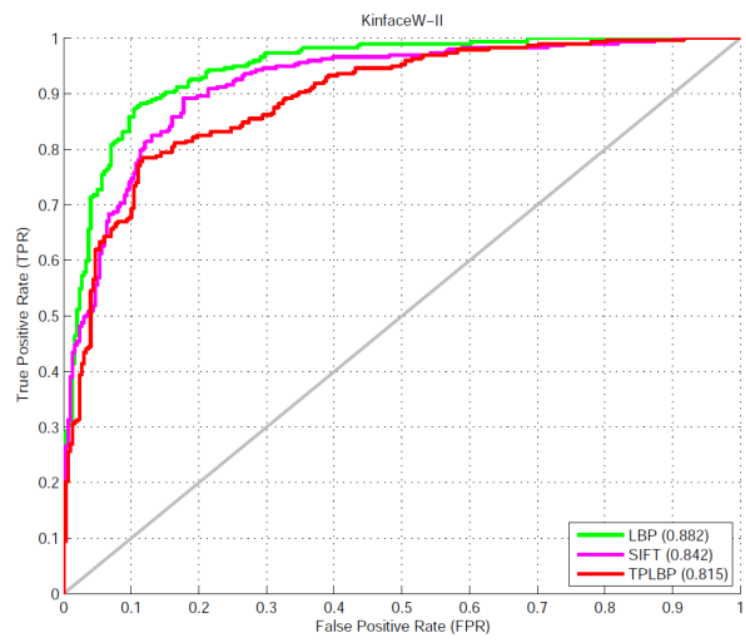

Figure 9. indicates the result of ROC of face descriptors on different datasets like KinfaceW-II with different possible relationships.

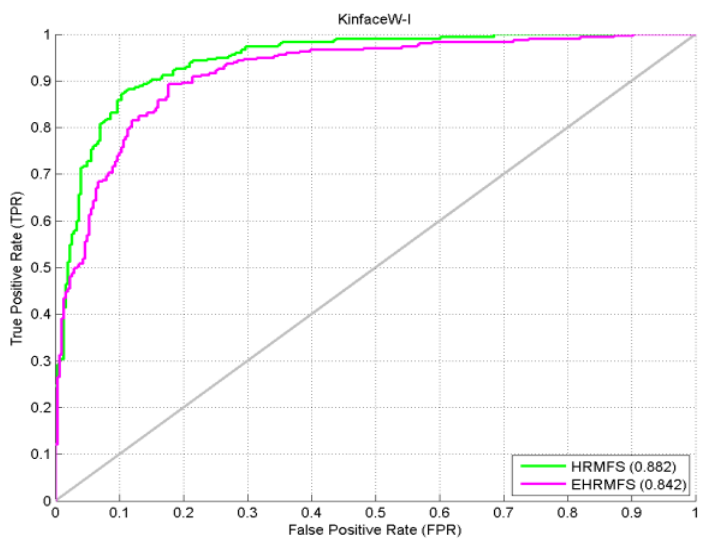

Figure 10. The accuracy of HRMFS and EHRMFS over KinfaceW-II dataset indicates the results of comparison 


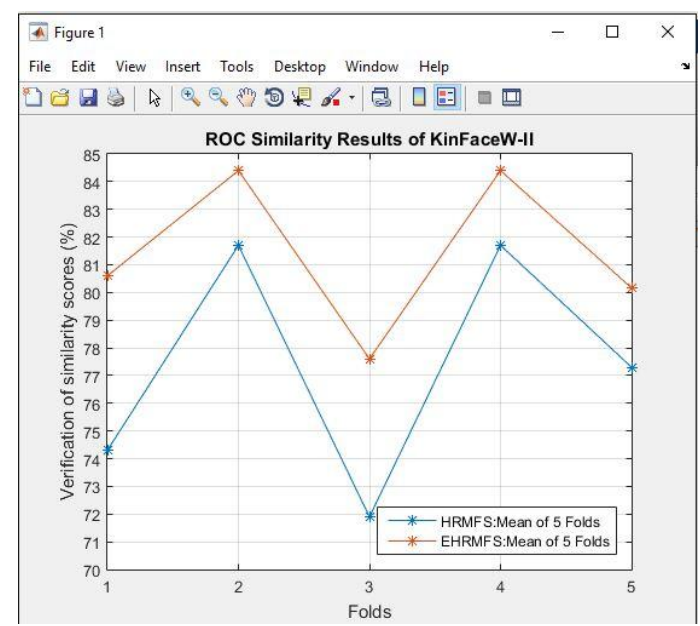

Figure 11. indicates the mean verification accuracy of the proposed method HRMFS and EHRMFS over a dataset KinfaceW-II.

Table 1. The Comparison of the proposed approach over NRML and MNRML approaches on KinfaceW-I and KinfaceW-II is tabulated

\begin{tabular}{|c|c|c|c|c|c|c|}
\hline Method & Feature & F-D & F-S & M-D & M-S & Mean \\
\hline CSML & LBP & 61.2 & 63.7 & 62.4 & 55.4 & 60.7 \\
\hline & LE & 58.1 & 61.1 & 70.0 & 60.9 & 62.5 \\
\hline & SIFT & 60.0 & 66.5 & 56.4 & 60.0 & 59.8 \\
\hline & TPLBP & 61.5 & 57.3 & 57.0 & 63.2 & 59.7 \\
\hline NCA & LBP & 62.2 & 61.7 & 62.4 & 56.4 & 60.7 \\
\hline & LE & 57.1 & 62.1 & 69.0 & 61.9 & 62.3 \\
\hline & SIFT & 61.0 & 67.5 & 57.4 & 61.0 & 60.8 \\
\hline & TPLBP & 60.5 & 56.3 & 56.0 & 62.2 & 58.7 \\
\hline & LBP & 63.2 & 62.7 & 63.4 & 57.4 & 61.7 \\
\hline & LE & 58.1 & 63.1 & 70.0 & 62.9 & 63.3 \\
\hline & SIFT & 63.0 & 69.5 & 59.4 & 63.0 & 62.8 \\
\hline NRRML & TPLBP & 61.5 & 57.3 & 57.0 & 63.2 & 59.7 \\
\hline & LBP & 65.2 & 64.7 & 65.4 & 59.4 & 63.7 \\
\hline & SIFT & 59.1 & 64.1 & 71.0 & 63.9 & 64.3 \\
\hline & TPLBP & 63.0 & $\mathbf{7 0 . 5}$ & 60.4 & 64.0 & 63.8 \\
\hline MNRML & All & 66.0 & 76.3 & 60.0 & 65.2 & 62.9 \\
\hline $\begin{array}{c}\text { Proposed } \\
\text { HRMFS }\end{array}$ & All & $\mathbf{8 1 . 7}$ & $\mathbf{7 1 . 9}$ & $\mathbf{7 4 . 8}$ & $\mathbf{7 4 . 8}$ & $\mathbf{7 6 . 9}$ \\
\hline $\begin{array}{c}\text { Proposed } \\
\text { EHRMFS }\end{array}$ & All & $\mathbf{8 4 . 4}$ & $\mathbf{7 7 . 6}$ & $\mathbf{8 0 . 6}$ & $\mathbf{8 0 . 6}$ & $\mathbf{8 0 . 2}$ \\
\hline
\end{tabular}


Table 2. The Comparison of the proposed approach over NRML and MNRML approaches on KinfaceW-II is tabulated

\begin{tabular}{|c|c|c|c|c|c|c|}
\hline Method & Feature & F-D & F-S & M-D & M-S & Mean \\
\hline \multirow[t]{4}{*}{ CSML } & LBP & 65.5 & 66.0 & 65.0 & 64.8 & 65.3 \\
\hline & LE & 68.1 & 71.8 & 74.0 & 73.8 & 71.9 \\
\hline & SIFT & 58.9 & 62.0 & 57.4 & 56.8 & 58.8 \\
\hline & TPLBP & 62.6 & 66.4 & 64.9 & 62.8 & 64.2 \\
\hline \multirow[t]{4}{*}{ NCA } & LBP & 66.5 & 67.0 & 66.0 & 65.8 & 66.3 \\
\hline & LE & 70.1 & 73.8 & 75.0 & 74.8 & 73.5 \\
\hline & SIFT & 59.9 & 63.0 & 59.4 & 58.8 & 60.4 \\
\hline & TPLBP & 63.6 & 67.4 & 66.9 & 63.8 & 66.5 \\
\hline \multirow[t]{4}{*}{ LMNN } & LBP & 68.5 & 68.0 & 67.0 & 68.8 & 68.2 \\
\hline & LE & 71.1 & 74.8 & 76.0 & 75.8 & 74.5 \\
\hline & SIFT & 57.9 & 65.0 & 59.4 & 58.8 & 60.4 \\
\hline & TPLBP & 65.6 & 68.4 & 67.9 & 65.8 & 68.1 \\
\hline \multirow[t]{4}{*}{ NRML } & LBP & 69.5 & 69.0 & 69.0 & 69.8 & 69.5 \\
\hline & LE & 73.1 & 76.8 & 77.0 & 76.8 & 75.7 \\
\hline & SIFT & 60.9 & 68.0 & 61.4 & 60.8 & 62.8 \\
\hline & TPLBP & 67.6 & 70.4 & 69.9 & 67.8 & 70.1 \\
\hline MNRML & All & 74.3 & 76.9 & 77.6 & 77.4 & 76.5 \\
\hline $\begin{array}{c}\text { Proposed } \\
\text { HRMFS }\end{array}$ & All & 74.3 & 81.7 & 71.9 & 81.7 & 77.3 \\
\hline $\begin{array}{c}\text { Proposed } \\
\text { EHRMFS }\end{array}$ & All & 80.6 & 84.4 & 77.6 & 84.4 & 80.16 \\
\hline
\end{tabular}

\subsection{Analysis of KinfaceW-I}

The proposed HRMFS and EHRMFS over a dataset KinfaceW-I gives detailed information in terms of efficiency in the form of numerical values as indicated in table III and table IV 
Table 3: Result of HRMFS over KinfaceW-I

\begin{tabular}{|c|c|c|c|c|}
\hline Folds & FD & FS & MD & MS \\
\hline $\mathbf{1}$ & 84.2 & 71.7 & 75.3 & 75.3 \\
\hline $\mathbf{2}$ & 81.9 & 74.1 & 75.0 & 75.0 \\
\hline $\mathbf{3}$ & 85.1 & 71.1 & 76.0 & 76.0 \\
\hline $\mathbf{4}$ & 82.9 & 71.3 & 77.1 & 77.1 \\
\hline $\mathbf{5}$ & 83.9 & 70.8 & 74.3 & 74.3 \\
\hline mean & 81.7 & 71.9 & 74.8 & 74.8 \\
\hline
\end{tabular}

\subsection{Analysis of KinfaceW-II}

The proposed HRMFS and EHRMFS and its efficiency over a KinfaceW-II dataset can be visualized in the form of numerical values as indicated in table $\mathrm{V}$ and table $\mathrm{VI}$

Table 5: Result of HRMFS over KinfaceW-II dataset Table VI: The result of EHRMFS over KinfaceW-II

\begin{tabular}{|c|c|c|c|c|}
\hline Folds & FD & FS & MD & MS \\
\hline $\mathbf{1}$ & 75.5 & 84.2 & 71.7 & 84.2 \\
\hline $\mathbf{2}$ & 74.3 & 76.9 & 74.1 & 76.9 \\
\hline $\mathbf{3}$ & 76.0 & 78.7 & 68.4 & 78.7 \\
\hline $\mathbf{4}$ & 76.0 & 83.0 & 72.6 & 83.0 \\
\hline $\mathbf{5}$ & 76.0 & 85.1 & 71.1 & 85.1 \\
\hline mean & $\mathbf{7 4 . 3}$ & $\mathbf{8 1 . 7}$ & $\mathbf{7 1 . 9}$ & $\mathbf{8 1 . 7}$ \\
\hline
\end{tabular}

\begin{tabular}{|c|c|c|c|c|}
\hline Folds & FD & FS & MD & MS \\
\hline $\mathbf{1}$ & 82.7 & 88.2 & 77.4 & 88.2 \\
\hline $\mathbf{2}$ & 80.7 & 81.6 & 78.3 & 81.6 \\
\hline $\mathbf{3}$ & 80.7 & 83.7 & 78.3 & 83.7 \\
\hline $\mathbf{4}$ & 83.3 & 84.4 & 79.0 & 84.4 \\
\hline $\mathbf{5}$ & 81.3 & 82.6 & 70.8 & 82.6 \\
\hline mean & $\mathbf{8 0 . 6}$ & $\mathbf{8 4 . 4}$ & $\mathbf{7 7 . 6}$ & $\mathbf{8 4 . 4}$ \\
\hline
\end{tabular}

\section{Conclusion}

The proposed work HRMFS and EHRMFS have been carried out using harmonic metric rule on a trained set of images of a dataset, it has given better results than other approaches in the domain of measuring facial similarity. The HRMFS and EHRMFS have yielded better results on a standard benchmark dataset, which can be understood from table I and table II.

\section{REFERENCE}

[1]. Jiwen lu, Xiuzhuang Zhou, Yap-Pen Tan, Yuanyuan Shang and Jie Zhou, "Neighborhood Repulsed Metric Learning for Kinship Verification," IEEE Transactions on Pattern Analysis and Machine Intelligence, vol. 36, no. 2, February 2014.

[2]. Tinne Tuytelaars and Krystian Mikolajczyk, "Local Invariant Feature Detectors: A Survey," Computer Graphics and Vision, Vol. 3, No. 3 (2007) 177-280, 2007.

[3]. Stefan Leutenegger, Margarita Chli and Roland Y. Siegwart Autonomous Systems Lab, "BRISK: Binary Robust Invariant Scalable Keypoints," 2011 International Conference on Computer Vision ,pp.2548- 2555, 6-13 November 2011.

[4]. Gwenae Kaminski, Slimane Dridi, Christian Graff and Edouard Gentaz, "Human ability to detect kinship in strangers faces: effects of the degree of relatedness," Proceedings of the royal society, doi:10.1098/rspb.2009.0677, 2009. 
Ravi Kumar Y B and C N Ravi Kumar; Harmonic Rule for Measuring the Facial Similarities among Relatives.

Transactions on Machine Learning and Artificial Intelligence, Volume 4 No 6 December (2016); pp: 29-49

[5]. Herbert Bay, Andreas Ess, Tinne Tuytelaars, Luc Van Gool, "Speeded- Up Robust Features (SURF)," Computer Vision and Image Understanding, 110 (2008) 346-359, 2008.

[6]. F. Tajeripour, E. Kabir, and A. Sheikhi, "Fabric Defect Detection Using Modified Local Binary Patterns," EURASIP Journal on Advances in Signal Processing, VOL.2008, Article ID 783898, 2008.

[7]. Jean-Michel Morel, Guoshen Yu, "Is SIFT Scale Invariant?," Inverse Problems and Imaging, Volume 5, No. $1,2011$.

[8]. Daniel Maturana, Domingo Mery and Alvaro Soto, "Learning Discriminative Local Binary Patterns for Face Recognition,"Automatic Face Gesture Recognition and Workshops (FG 2011),pp. 470-475, 21-25 March 2011.

[9]. Sebastian Hegenbart, Andreas Uhl, Andreas Vecsei, "An Affine Invariant Local Ternary Pattern," Technical Report, 2013-03 July 2013.

[10]. Bassem Sheta, Mohamed Elhabiby, and Naser El-Sheimy, "Assessments of different Speeded up Robust Features (SURF) Algorithm Resolution for Pose Estimation of UAV," International Journal of Computer Science Engineering Survey (IJCSES),Vol.3, No.5, October 2012.

[11]. Baochang Zhang, Yongsheng Gao, Sanqiang Zhao, and Jianzhuang Liu, "Local Derivative Pattern Versus Local Binary Pattern: Face Recognition With High Order Local Pattern Descriptor," IEEE Transactions of Image Processing,VOL. 19, NO. 2, FEBRUARY 2010.

[12]. Min Xu and Yuanyuan Shang, "Kinship Verification Using Facial Images by Robust Similarity Learning," Hindawi Publishing Corporation Mathematical Problems in Engineering, Volume 2016, Article ID 4072323, 8 pages, Junuary 2016.

[13]. Zhenhua Guo, Lei Zhang, and David Zhang, "Adaptive Extended Local Ternary Pattern (AELTP) for Recognizing Avatar Faces," 11th International Conference on Machine Learning and Applications, IEEE, DOI 10.1109/ICMLA.2012.19, 2012.

[14]. Stefanie Jegelka, Arthur Gretton, “Brisk Kernel ICA,”MIT Press, pp.225-250, 2007.

[15]. Mauro Franceschelli, Andrea Gasparri, Alessandro Giua, Carla Seatzu, "Decentralized Estimation of Laplacian Eigenvalues in Multi-Agent Systems," Automatica, vol. 49, num. 4, pages 1031-1036, 2013.

[16]. Haibin Yan, Jiwen Lu, Weihong Deng, and Xiuzhuang Zhou, "Discriminative Multimetric Learning for Kinship Verification," IEEE Transactions on Information Forensics and Security, VOL. 9, NO. 7, JULY 2014.

[17]. Bradley W. Dickinson, and Kenneth Steiglitz, "Eigenvectors and Functions of the Discrete Fourier Transform," IEEE TRANSACTIONS ON ACOUSTICS, SPEECH, AND SIGNAL PROCESSING,VOL. ASSP-30, NO. 1 , FEBRUARY 1982.

[18]. Loris Nanni, Sheryl Brahnam, Alessandra Lumini, "Selecting the best performing rotation invariant patterns in local binary/ternary patterns," Int'l Conf. IP, Comp. Vision, and Pattern Recognition, IPCV'10.,pp.369-374,2010. 
[19]. Herbert Bay, Andreas Ess, Tinne Tuytelaars, and Luc Van Gool, "Speeded-Up Robust Features (SURF)," Computer Vision and Image Understanding, VOL 110, pp. 346-359, June 2008.

[20]. Mayank Agarwal, Nikunj Jain, Mr. Manish Kumar and Himanshu Agrawal, “Face Recognition Using Eigen Faces and Artificial Neural Network," International Journal of Computer Theory and Engineering,Vol. 2, No. 4, 1793-8201,August, 2010.

[21]. Xiuzhuang Zhou, Jiwen Lu, Junlin Hu, Yuanyuan Shang, "Gabor-Based Gradient Orientation Pyramid for Kinship Verification Under Uncontrolled

[22]. Environments MM'12,"October 29-November 2, 2012.

[23]. David G. Lowe, "Object Recognition from Local Scale-Invariant Features," Proc. of the International Conference on Computer Vision,Corfu(Sept. 1999).

[24]. Siyu Xia, Ming Shao, Jiebo Luo, and Yun Fu, "Understanding Kin Relationships in a Photo,"IEEE TRANSACTIONS ON MULTIMEDIA, VOL. 14, NO. 4, AUGUST 2012.

[25]. Junlin Hu, Jiwen Lu, Junsong Yuan, Yap-Peng Ta, "Large Margin Multi-Metric Learning for Face and Kinship Verification in the Wild,"Springer International Publishing, Switzerland, 2015,ACCV 2014,Part III, LNCS 9005, pp.252-267,2015.

[26]. David G. Lowe, "Distinctive Image Features from Scale-Invariant Keypoints," International Journal of Computer Vision,VOL. 60, pp.91-110, 1 November 2004.

[27]. Haibin Yan, Jiwen Lu, and Xiuzhuang Zhou, "Prototype-Based Discriminative Feature Learning for Kinship Verification,"IEEE Transactions on cybernetics, VOL 45, pp.2535-2545, 10 December 2014.

[28]. Zhenhua Guo, Lei Zhang, David Zhang, "Rotation invariant texture classification using LBP variance (LBPV) with global matching," Pattern Recognition, VOL 43, pp.706-719, 2010.

[29]. Xiuzhuang Zhou, Junlin Hu, Jiwen Lu, Yuanyuan Shang, Yong Guan, "Kinship Verification from Facial Images Under Uncontrolled Conditions," ACM, November 28-December 1, 2011.

[30]. Xiaoyang Tan and Bill Triggs, "Enhanced Local Texture Feature Sets for Face Recognition under Difficult Lighting Conditions," IEEE Transactions on Image Processing, pp. 1635-1650, 17 February 2010.

[31]. Lilei Zheng, Khalid Idrissi, Christophe Garcia, Stefan Duffner and Atilla Baskurt, "Triangular Similarity Metric Learning for Face Verification,", VOL 1, pp.1-7, 4-8 May 2015. 